\title{
Hyper-Ramsey Spectroscopy of Optical Clock Transitions
}

\author{
V. I. Yudin and A. V. Taichenachev \\ Institute of Laser Physics SB RAS, Novosibirsk 630090, Russia \\ Novosibirsk State University, Novosibirsk 630090, Russia and \\ Novosibirsk State Technical University, Novosibirsk 630092, Russia \\ C. W. Oates, Z. W. Barber, N. D. Lemke, and A. D. Ludlow \\ National Institute of Standards and Technology, 325 Broadway, Boulder, CO 80305, USA \\ U. Sterr, Ch. Lisdat, and F. Riehle \\ Physikalisch-Technische Bundesanstalt (PTB), Bundesallee 100, 38116 Braunschweig, Germany
}

(Dated: May 28, 2018)

\begin{abstract}
We present non-standard optical Ramsey schemes that use pulses individually tailored in duration, phase, and frequency to cancel spurious frequency shifts related to the excitation itself. In particular, the field shifts and their uncertainties of Ramsey fringes can be radically suppressed (by 2-4 orders of magnitude) in comparison with the usual Ramsey method (using two equal pulses) as well as with single-pulse Rabi spectroscopy. Atom interferometers and optical clocks based on two-photon transitions, heavily forbidden transitions or magnetically induced spectroscopy could significantly benefit from this method. In the latter case these frequency shifts can be suppressed considerably below a fractional level of $10^{-17}$. Moreover, our approach opens the door for the high-precision optical clocks based on direct frequency comb spectroscopy.
\end{abstract}

PACS numbers: 03.75.Dg, 06.20.F-, 37.25.+k, 42.62.Fi

Presently laser spectroscopy and fundamental metrology are among of the most important and actively developed directions in modern physics. Frequency and time are the most precisely measured physical quantities, which, apart from practical applications (in navigation and information systems), play critical roles in tests of fundamental physical theories (such as QED, QCD, unification theories, cosmology etc.) 1]. Now laser metrology is confronting the challenging task of creating an optical clock with fractional inaccuracy and instability at the level of $10^{-17}-10^{-18}$. Indeed, considerable progress has already been achieved along this path for both ion-trap [2] and atomic lattice-based [3, 4] clocks.

Work in this direction has stimulated the development of novel spectroscopic methods (e.g., spectroscopy using quantum logic [5] and magnetically induced spectroscopy [6]). For some of the promising clock systems, one of the key unsolved problems is the frequency shift of the clock transition due to the excitation pulses themselves. For the case of magnetically induced spectroscopy these shifts (quadratic Zeeman and ac-Stark shifts) could ultimately limit the achievable performance. Moreover, for ultra-narrow transitions (e.g., electric octupole 7] and two-photon transitions [8, 9]) the ac-Stark shift can be so large in some cases to rule out high accuracy clock performance at all. A similar limitation exists for clocks based on direct frequency comb spectroscopy [10, 11] due to ac-Stark shifts induced by large numbers of off-resonant laser modes.

In this paper we propose a general solution to this important problem, which is based on the development and generalization of the Ramsey method [12]. We have found that in contrast with the single-pulse (Rabi) technique, multi-pulse Ramsey spectroscopy offers several ways (e.g., pulse durations, frequencies, and phases) to significantly manipulate (due to interference effects) induced frequency shifts of the spectroscopy signals. In particular, for special excitation schemes (which we refer to as "hyper-Ramsey") the resulting ac-Stark shift depends on the laser intensity in an essentially non-linear way. Such unusual and unexpected behavior allows us to dramatically suppress these shifts and their uncertainties (most critical for clocks) by two to four orders of magnitude with strongly relaxed control requirements for the experimental parameters. Additionally, we have found that these schemes can have a greatly reduced sensitivity to the pulse areas, which makes the procedure robust and accessible experimentally. Thus, this method can be readily implemented (as needed) in a variety of existing and proposed clock systems [2 11, 13]. Our approach could lead to significant progress for atomic clocks: it will improve several key existing optical clock systems, and could enable new systems that were not previously thought to be competitive.

Note, some variants of the Ramsey technique were proposed in 13, 14] to cancel the overall field shift. However, the shift uncertainties (caused by the fluctuations of field parameters) were not significantly reduced in [13, 14], as it is possible with the hyper-Ramsey method.

The hyper-Ramsey spectroscopy schemes (Fig. 11) are based on time-separated pulses that can have different durations, frequencies, and phases. The action of a single light pulse (with frequency $\omega_{\mathrm{p}}$, duration $\tau$, and Rabi frequency $\Omega_{0}$ ) on two-level atoms with ground and ex- 


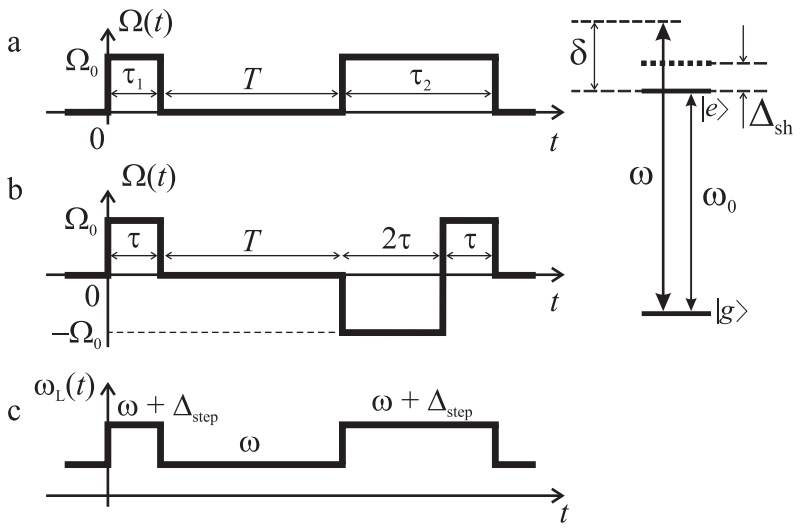

FIG. 1: Ramsey pulses with Rabi frequency $\Omega_{0}$ of different duration $\left(\tau_{1}\right.$ and $\tau_{2}$; panel (a)) and with a phase step in the second pulse $\left(\tau_{2}=3 \tau_{1}\right.$; panel (b)). During the pulses, we step the laser frequency $\omega$ by $\Delta_{\text {step }}$ (panel (c) and text). Also shown is a two-level atom with splitting $\omega_{0}$, detuning $\delta$ of the laser with frequency $\omega$ during dark time $T$, and excitation related shift $\Delta_{\text {sh }}$ during pulses.

cited states, $|g\rangle=\left(\begin{array}{l}0 \\ 1\end{array}\right)$ and $|e\rangle=\left(\begin{array}{l}1 \\ 0\end{array}\right)$ (separated by the unperturbed energy $\left.\hbar \omega_{0}\right)$, is described by the matrix:

$$
\begin{aligned}
& \widehat{W}\left(\tau, \Omega_{0}, \delta_{\mathrm{p}}\right)= \\
& \left(\begin{array}{cc}
\cos \left(\frac{\Omega \tau}{2}\right)+\frac{i \delta_{\mathrm{p}}}{\Omega} \sin \left(\frac{\Omega \tau}{2}\right) & \frac{i \Omega_{0}}{\Omega} \sin \left(\frac{\Omega \tau}{2}\right) \\
\frac{i \Omega_{0}}{\Omega} \sin \left(\frac{\Omega \tau}{2}\right) & \cos \left(\frac{\Omega \tau}{2}\right)-\frac{i \delta_{\mathrm{p}}}{\Omega} \sin \left(\frac{\Omega \tau}{2}\right)
\end{array}\right),
\end{aligned}
$$

where $\Omega=\sqrt{\Omega_{0}^{2}+\delta_{\mathrm{p}}^{2}}$ is the generalized Rabi frequency. The detuning during pulse $\delta_{\mathrm{p}}=\omega_{\mathrm{p}}-\omega_{0}-\Delta_{\mathrm{sh}}$ contains the excitation related shift $\Delta_{\text {sh }}$ (see Fig. 1, level scheme) due to the influence of other (far-off-resonant) transitions. Within the frequency interval corresponding to the narrow clock resonance the variation of $\Delta_{\mathrm{sh}}$ on $\omega_{\mathrm{p}}$ is negligible, i.e., $\Delta_{\text {sh }}$ is a constant (for fixed $\Omega_{0}$ ).

During the dark period between the pulses, excitation related shifts (which produce the total actual shift $\Delta_{\mathrm{sh}}$ ) are absent (e.g., the ac-Stark shift from the laser) or can be turned off (like the Zeeman shift). If during the dark period $T$ the laser frequency is $\omega$, then the free evolution is described by the matrix with detuning $\delta=\omega-\omega_{0}$ :

$$
\widehat{V}(T \delta)=\left(\begin{array}{cc}
e^{i T \delta / 2} & 0 \\
0 & e^{-i T \delta / 2}
\end{array}\right) .
$$

In the general case, the laser frequency during the pulse does not have to be the same as the frequency during the dark time, i.e., $\omega_{\mathrm{p}} \neq \omega[14]$. As we will see, at times it can be useful to approximately offset the induced shift, $\Delta_{\mathrm{sh}}$, by stepping the laser frequency only during the pulses by a fixed $\Delta_{\text {step }}$, i.e., $\omega_{\mathrm{p}}=\omega+\Delta_{\text {step }}$ (see Fig. 1r). Thus, in the general case the detuning during the pulses can be written as $\delta_{\mathrm{p}}=\delta-\Delta$, where $\Delta=\Delta_{\text {sh }}-\Delta_{\text {step }}$ is the effective frequency shift (during the pulse).

If at $t=0$ atoms are in the lower level $|g\rangle$, then after the action of two pulses of duration $\tau_{1}$ and $\tau_{2}$ separated by dark period $T$ (see Fig. 1a) the population $n_{\mathrm{e}}$ of atoms in the excited state is determined by

$$
n_{\mathrm{e}}=\left|\left\langle e\left|\widehat{W}\left(\tau_{2}, \Omega_{0}, \delta-\Delta\right) \widehat{V}(T \delta) \widehat{W}\left(\tau_{1}, \Omega_{0}, \delta-\Delta\right)\right| g\right\rangle\right|^{2} .
$$

Equation (3) describes Ramsey fringes (as a function of variable detuning $\delta$, but with fixed $\Delta$ ). The presence of the additional shift $\Delta$ in the course of the pulse action leads to a shift $\overline{\delta \omega}_{0}$ of the position (top or bottom) of the central Ramsey fringe with respect to the unperturbed frequency $\omega_{0}$. To investigate the dependence of $\overline{\delta \omega}_{0}$ on $\Delta$ we present the signal $n_{\mathrm{e}}$ as a Taylor expansion in terms of the dimensionless parameter $(T \delta)$ :

$$
n_{\mathrm{e}}=a^{(0)}+a^{(1)}(T \delta)+a^{(2)}(T \delta)^{2}+\ldots .
$$

The coefficients $a^{(j)}$ are expanded in the powers of $\Delta / \Omega_{0}$ :

$$
\begin{aligned}
& a^{(0)}=\mathcal{A}_{0}^{(0)}+\mathcal{A}_{2}^{(0)}\left(\Delta / \Omega_{0}\right)^{2}+\mathcal{A}_{4}^{(0)}\left(\Delta / \Omega_{0}\right)^{4}+\ldots \\
& a^{(1)}=\mathcal{A}_{1}^{(1)}\left(\Delta / \Omega_{0}\right)+\mathcal{A}_{3}^{(1)}\left(\Delta / \Omega_{0}\right)^{3}+\ldots \\
& a^{(2)}=\mathcal{A}_{0}^{(2)}+\mathcal{A}_{2}^{(2)}\left(\Delta / \Omega_{0}\right)^{2}+\mathcal{A}_{4}^{(2)}\left(\Delta / \Omega_{0}\right)^{4}+\ldots
\end{aligned}
$$

The occurrence of terms with only odd or even powers is the direct consequence of the symmetry of Eq. (3) that does not change under the simultaneous substitutions $\delta \rightarrow-\delta$ and $\Delta \rightarrow-\Delta$, which holds for any sequence of pulses described by the matrices $\widehat{W}$ and $\widehat{V}$.

Even if the actual level shift $\Delta_{\text {sh }}$ is comparable to or larger than $\Omega_{0}$, we can always apply a frequency step $\Delta_{\text {step }}$ (e.g., with an acousto-optic modulator) during excitation to achieve the condition $\left|\Delta / \Omega_{0}\right| \ll 1$ for an effective shift $\Delta . \Delta_{\text {step }}$ can be evaluated experimentally by variation of the dark period $T$. If $\Delta_{\text {step }} \neq \Delta_{\text {sh }}$, the observed transition frequency will be dependent on $T$ [14]. With a control of the shift to $1 \%$ under typical conditions we can achieve $\left|\Delta / \Omega_{0}\right|<0.01$ to 0.1 .

Under the condition $\left|\Delta / \Omega_{0}\right| \ll 1$ we use the parabolic approximation in Eq. (4) (on $|T \delta| \ll 1$ ) to find the leading approximation of $\overline{\delta \omega}_{0}$ :

$$
\overline{\delta \omega_{0}} \approx-\frac{1}{T} \frac{a^{(1)}}{2 a^{(2)}} .
$$

With Eq. (5), the dominant dependence of $\overline{\delta \omega}_{0}$ on $\Delta / \Omega_{0}$ can be identified. For the usual Ramsey scheme with $\tau_{1} \Omega_{0}=\tau_{2} \Omega_{0}=\pi / 2$ we find the expected linear dependence:

$$
\overline{\delta \omega}_{0} \approx-\frac{1}{T} \frac{\mathcal{A}_{1}^{(1)}}{2 \mathcal{A}_{0}^{(2)}}\left(\frac{\Delta}{\Omega_{0}}\right)=\frac{1}{T} \frac{2 \Omega_{0} T}{2+\Omega_{0} T} \frac{\Delta}{\Omega_{0}} .
$$

Explicit analytical calculations for the first term $\mathcal{A}_{1}^{(1)}$ in the expansion of $a^{(1)}$ of Eq. (5) show that

$$
\mathcal{A}_{1}^{(1)} \propto \sin \left[\Omega_{0}\left(\tau_{1}+\tau_{2}\right) / 2\right] .
$$



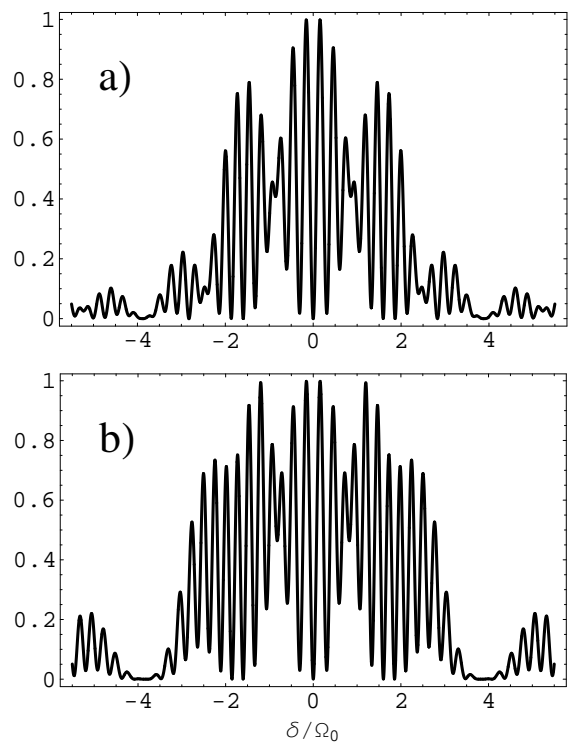

FIG. 2: Hyper-Ramsey fringes $n_{\mathrm{e}}(\delta)$ under the conditions $\Omega_{0} \tau_{1}=\pi / 2, \tau_{2} / \tau_{1}=3, \Omega_{0} T=20, \Delta=0$ according to a) Eq. (3) and b) Eq. (13).

Thus, we find that $\mathcal{A}_{1}^{(1)}=0$ for

$$
\Omega_{0}\left(\tau_{1}+\tau_{2}\right)=2 \pi n \quad(n=1,2,3, \ldots) .
$$

From Eqs. (5) and (6), we see that for $\mathcal{A}_{1}^{(1)}=0$ and $\mathcal{A}_{0}^{(2)} \neq 0$, the dominating dependence of $\overline{\delta \omega}_{0}$ on $\left|\Delta / \Omega_{0}\right| \ll 1$ is now cubic:

$$
\overline{\delta \omega}_{0} \approx-\frac{1}{T} \frac{\mathcal{A}_{3}^{(1)}}{2 \mathcal{A}_{0}^{(2)}}\left(\frac{\Delta}{\Omega_{0}}\right)^{3} .
$$

Equation (10) has two important consequences. First, under the condition $\left|\Delta / \Omega_{0}\right| \ll 1$, the resulting shift of the central hyper-Ramsey fringe is much smaller than for the usual Ramsey scheme (Eq. (77)). Second, it has a higher-order dependence, which is the reason for referring to this method as the "hyper-Ramsey" method.

Apart from the condition Eq. (9), which essentially minimizes the shift $\overline{\delta \omega}_{0}$, for applications in spectroscopy and optical clocks it is also desirable to maximize the amplitude of the central resonance. This is accomplished by the maximization of the coefficient $\mathcal{A}_{0}^{(2)}$ in Eq. (5), which defines the curvature of the central resonance top if $\left|\Delta / \Omega_{0}\right| \ll 1$. Under the condition of Eq. (9) we find $\mathcal{A}_{0}^{(2)}=0.25 \sin ^{2}\left(\Omega_{0} \tau_{1}\right)$. Then, the coefficient $\mathcal{A}_{0}^{(2)}$ reaches its maximum at

$$
\Omega_{0} \tau_{1}=\pi(2 m+1) / 2 \quad(m=0,1,2, \ldots) .
$$

Equations (9) and (11) lead to the following relationship for the values $\tau_{1}$ and $\tau_{2}$ :

$$
\tau_{2} / \tau_{1}=(4 n-2 m-1) /(2 m+1) .
$$

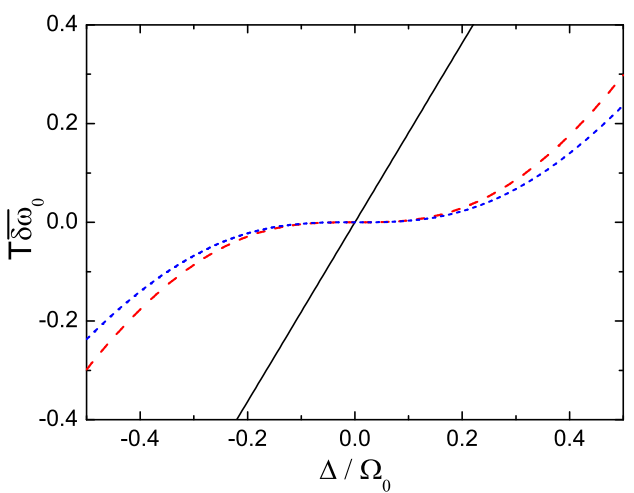

FIG. 3: (color) Numerically calculated shift of the central resonance $T \overline{\delta \omega}_{0}$ versus $\Delta / \Omega_{0}$ for standard Ramsey spectroscopy $\left(\Omega_{0} \tau_{1}=\Omega_{0} \tau_{2}=\pi / 2 ; \Omega_{0} T=20 ;\right.$ full line $)$ and the hyper-Ramsey method $\left(\Omega_{0}\left(\tau_{1}+\tau_{2}\right)=2 \pi ; \tau_{2} / \tau_{1}=3 ; \Omega_{0} T=20\right)$. Dashed line: position of maximum; dotted line: estimate of center from signal comparison with $\pm \pi / 2$ phase steps [15].

In the simplest case $n=1$ (i.e., when $\Omega_{0}\left(\tau_{1}+\tau_{2}\right)=2 \pi$ ) we find that either $\tau_{2} / \tau_{1}=3$ or $\tau_{2} / \tau_{1}=1 / 3$. The signal contrast is close to the maximum value of 1 (see Fig. 2 $2 \mathrm{a}$ ). The coefficient of the cubic term of Eq. (10) in the case of $T \gg\left(\tau_{1}+\tau_{2}\right)$ then amounts to $\mathcal{A}_{3}^{(1)} / 2 \mathcal{A}_{0}^{(2)} \approx-\pi$.

The elimination of the shift $\Delta$ from the observed resonance shift $\overline{\delta \omega}_{0}$ is illustrated in Fig. 3through comparison with the regular Ramsey method using Eq. (3). For the hyper-Ramsey case, we find the residual frequency shift in two ways: by locating the maximum of the fringe and by generating a discriminator slope at zero detuning $\delta$ by stepping the phase of one of the pulses by $\pm \pi / 2$ in an alternating way [15] and equalizing these signals. The latter approach is of greater relevance for clocks, because it directly generates an error signal with high sensitivity. However, probing the interference at the half width points introduces a weak linear (on $\Delta / \Omega_{0}$ ) contribution for $\overline{\delta \omega}_{0}$ in the hyper-Ramsey signal.

In some cases such as that for lattice-based and single ion clocks, we cannot perfectly fulfill condition Eq. (9) even with good intensity control, e.g., due to vibrational state-dependent Rabi frequencies. In Fig. 4 we plot the resultant $\overline{\delta \omega}_{0}$ versus $\left(\Delta / \Omega_{0}\right)$ dependence for non-optimal values of the sum $\tau_{1}+\tau_{2}$. Here we see how a deviation from $\Omega_{0}\left(\tau_{1}+\tau_{2}\right)=2 \pi$ re-introduces the linear dependence on $\Delta / \Omega_{0}$, though still with a strongly reduced amplitude compared to that for usual Ramsey spectroscopy.

However, this inherent problem can be easily overcome by introducing a phase jump of $\pi$ at the beginning of the second pulse of the hyper-Ramsey sequence (Fig. 1b). The phase is stepped back after $2 / 3$ of the second pulse, such that the second Ramsey pulse can be seen as being composed of a pulse with $-\Omega_{0}$ (echo pulse) directly followed by one with $\Omega_{0}$. With modern electronic oscil- 


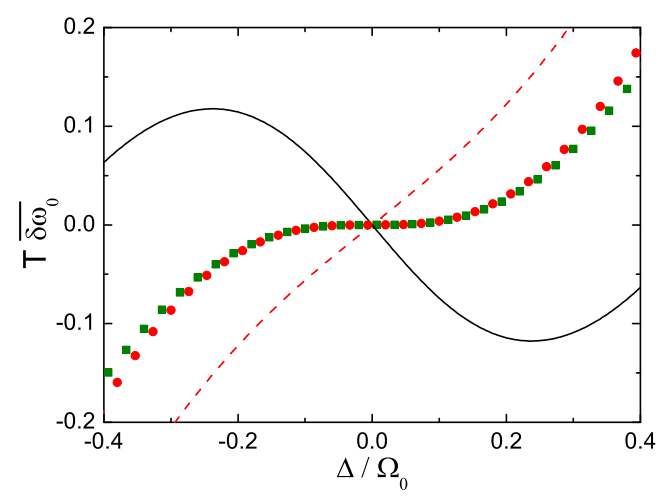

FIG. 4: (color) Influence of excitation pulse area on shift suppression (in calculations we used the approach [15] to find the center from signal comparison with $\pm \pi / 2$ phase steps for the second Ramsey pulse). Lines (solid and dotted) show numerically calculated maximum and minimum shifts $T \overline{\delta \omega}_{0}$ of the central fringe versus $\Delta / \Omega_{0}$ (the normalized effective frequency shift) for the hyper-Ramsey excitation in Fig. 1 1 for a range of the pulse area parameter, $\Omega_{0} \tau_{1}=q \pi / 2\left(\tau_{2} / \tau_{1}=3 ; \Omega_{0} T=20\right)$ of $0.9 \leq q \leq 1.1$. These lines show how the hyper-Ramsey suppression effect is compromised by non-optimized pulse areas. The symbols (squares and circles) show results for the hyper-Ramsey scheme in Fig. 10 (i.e. with additional $\pi$ phase jumps) also for $\Omega_{0} \tau=q \pi / 2\left(\Omega_{0} T=20\right)$ and $0.9 \leq q \leq 1.1$. In this case the suppression is largely insensitive to total pulse area, making this technique more feasible experimentally.

lators, namely direct digital synthesizers, it is easily possible to maintain phase coherence over both frequency steps and phase jumps. Note, the second pulse for the excitation in Fig. 10 has a technical similarity to the composite pulses used in NMR spectroscopy [16.

The expression for the population $n_{\mathrm{e}}$ can be extended to include the phase step in Fig. 1 b and we then find:

$$
\begin{aligned}
& n_{\mathrm{e}}= \\
& \left|\left\langle e\left|\widehat{W}\left(\tau, \Omega_{0}, \delta_{\mathrm{p}}\right) \widehat{W}\left(2 \tau,-\Omega_{0}, \delta_{\mathrm{p}}\right) \widehat{V}(T \delta) \widehat{W}\left(\tau, \Omega_{0}, \delta_{\mathrm{p}}\right)\right| g\right\rangle\right|^{2},
\end{aligned}
$$

where $\tau$ is the duration of the short pulse. In this case $\mathcal{A}_{1}^{(1)}=0$, i.e., the cubic dependence of Eq. (10) applies for arbitrary values $\Omega_{0}$ and $\tau$. Under conditions $\Omega_{0} \tau=\pi / 2$ and $T \gg 4 \tau$ we then find that $\mathcal{A}_{3}^{(1)} / 2 \mathcal{A}_{0}^{(2)} \approx-4$. The curves in Fig. 4 were calculated from Eq. (13) using $\Omega_{0} \tau \approx \pi / 2$ to maximize $(\sim 1)$ the signal contrast (see Fig. 2b). They show the large advantage of adding the phase step to the hyper-Ramsey sequence in terms of suppressing the dependence on pulse area.

Let us consider a numerical example using magnetically induced spectroscopy of ${ }^{174} \mathrm{Yb}[6,17]$. Since the advantage of the hyper-Ramsey method over usual Ramsey spectroscopy is obvious (by $2-4$ orders for $\left|\Delta / \Omega_{0}\right|<0.1$ 0.01 ), here we compare hyper-Ramsey to Rabi spectroscopy. If we assume typical experimental conditions of $T=40 \mathrm{~ms}, \tau=10 \mathrm{~ms}\left(\Omega_{0} / 2 \pi=25 \mathrm{~Hz}\right)$ at a magnetic field of $2 \mathrm{mT}$, an ac-Stark shift of $70 \mathrm{~Hz}$ (during pulses) will result. If we further assume that we can control the intensity to $1 \%$, we should be able to zero the effective detuning with an uncertainty of $\Delta / 2 \pi \approx 0.7 \mathrm{~Hz}$. The resulting suppression inherent in the technique then leaves a shift (and resultant uncertainty) of $0.35 \mathrm{mHz}$ (the fractional level is below of $10^{-18}$ for $518 \mathrm{THz}$ clock transition) for the central fringe, which is a factor of 120 less than the uncertainty for the corresponding Rabi case (with $1 \%$ control on a $4.2 \mathrm{~Hz}$ shift for a $80 \mathrm{~ms} \pi$-pulse).

Thus, the hyper-Ramsey method is a novel technique that offers a spectroscopic signal that is virtually free from excitation-induced frequency shifts and their fluctuations. It is robust against perturbations and noise, because the influence of the shifts on the signal is essentially eliminated. This method will now allow a variety of systems to enjoy other advantages of Ramsey spectroscopy: increased resolution for a given interrogation time, and improved stability [18, 19]. The hyperRamsey method has broad applications for optical clocks, especially those based on ultra-narrow transitions, twophoton transitions, or lattice clocks based on bosonic isotopes with controlled collision shifts [3, 20]. Moreover, our approach opens a prospect for the high-precision optical clocks based on direct frequency comb spectroscopy. High resolution matter-wave sensors 21] are also expected to benefit from the suppression of phase shifts in the interference patterns due to the excitation pulses.

We thank Ch. Tamm, E. Peik, T. Mehlstäubler and T. Heavner for useful discussions. A.V.T. and V.I.Yu. were supported by RFBR (08-02-01108, 10-02-00591, 1008-00844) and programs of RAS. V.I.Yu., U.S., Ch.L. and F.R. acknowledge gratefully support by the Center for Quantum Engineering and Space-Time Research (QUEST), the European Community's ERA-NET-Plus Programme under Grant Agreement No. 217257, and by the ESA and DLR in the project Space Optical Clocks.

V.I.Yudin e-mail address: viyudin@mail.ru

[1] S. A. Diddams et al., Science 306, 1318 (2004).

[2] T. Rosenband et al., Science 319, 1808 (2008).

[3] T. Akatsuka, M. Takamoto, and H. Katori, Nature Physics 4, 954 (2008).

[4] A. D. Ludlow et al., Science 319, 1805 (2008).

[5] P. O. Schmidt et al., Science 309, 749 (2005).

[6] Z. W. Barber et al., Phys. Rev. Lett. 96, 083002 (2006).

[7] K. Hosaka et al., Phys. Rev. A 79, 033403 (2009).

[8] M. Fischer et al., Phys. Rev. Lett. 92, 230802 (2004).

[9] T. Badr et al., Phys. Rev. A 74, 062509 (2006).

[10] T. M. Fortier et al., Phys. Rev. Lett. 97, 163905 (2006).

[11] M. C. Stowe et al., Advances in atomic, molecular and optical physics 55, 2 (2008).

[12] N. F. Ramsey, Phys. Rev. 78, 695 (1950).

[13] T. Zanon-Willette et al., Phys. Rev. Lett. 97, 233001 
(2006).

[14] A. V. Taichenachev et al., JETP Lett. 90, 713 (2009).

[15] A. Morinaga et al., Appl. Phys. B 48, 165 (1989).

[16] M. H. Levitt, in Encyclopedia of Nuclear Magnetic Resonance, edited by D. M. Grant and R. K. Harris (John Wiley \& Sons, Chichester, 1996), Vol. 2, pp. 1396-1411.

[17] A. V. Taichenachev et al., Phys. Rev. Lett. 96, 083001 (2006).

[18] G. Dick, in Proceedings of $19^{\text {th }}$ Annu. Precise Time and
Time Interval Meeting, Redendo Beach, 1987 (U.S. Naval Observatory, Washington, DC, 1988), pp. 133-147.

[19] A. Quessada et al., J. Opt. B: Quantum Semiclass. Opt. 5, S150 (2003).

[20] C. Lisdat et al., Phys. Rev. Lett. 103, 090801 (2009).

[21] F. Yver-Leduc et al., J. Opt. B: Quantum Semiclass. Opt. 5, S136 (2003). 